\title{
Fixed $p-i-n$ junction polymer light-emitting electrochemical cells based on charged self-assembled monolayers
}

\author{
D. T. Simon, D. B. Stanislowski, and S. A. Carter ${ }^{\text {a) }}$ \\ Physics Department, University of California, Santa Cruz, 1156 High Street, Santa Cruz, California 95064
}

(Received 4 January 2007; accepted 1 February 2007; published online 6 March 2007)

\begin{abstract}
The authors report on enhanced efficiency of polymer light-emitting electrochemical cells (LECs) by means of forming a $n$-doping self-assembled monolayer (SAM) at the cathode-polymer interface. The addition of the SAM, a silane-based salt with structural similarity to the commonly used LEC $n$-dopant tetra- $n$-butylammonium, caused a twofold increase in quantum efficiency. Photovoltaic analysis indicates that the SAM increases both the open-circuit voltage and short-circuit current. Current versus voltage data are presented which indicate that the SAM does not simply introduce an interfacial dipole layer, but rather provides a fixed doping region, and thus a more stable $p-i-n$ structure. (C) 2007 American Institute of Physics. [DOI: 10.1063/1.2711769]
\end{abstract}

Polymer light-emitting electrochemical cells (LECs) have been given much attention in recent years as an enhancement over the simpler polymer light-emitting diode (LED) ${ }^{1-3}$ These devices differ from the standard polymer LED structure ${ }^{4,5}$ in that the active polymer layer is blended with "doping" counterions and a solid polymer electrolyte. When a bias is applied across the device, charge is created within the polymer by oxidation/reduction of the conjugated backbone. The counterions migrate toward the electrodes and stabilize ("dope") the polymer charge, thereby creating $p$ - and $n$-doped regions at the anode and cathode, respectively, and leaving a relatively undoped intrinsic region in the middle. The resulting $p-i-n$ structure has the advantage over the standard polymer LED of higher conductivity at the doped interfaces, enabling a larger amount of charge to be injected and radiatively recombine in the central insulating region.

The trade-off for this enhancement is that it takes time to move the ions and establish the $p-i-n$ junction.$^{6-8}$ Furthermore, when the bias is removed, the ions forming the junction migrate away from the interfaces and back toward a neutral position within the bulk of the device. For this reason, several methods have been devised to stabilize the junction in the absence of bias, among them, "frozen" junctions, ${ }^{6,9,10}$ where the $p-i-n$ structure is formed at elevated temperature and then fixed at suppressed temperature, and additional polymer electrolyte/counterion layers near the interfaces ${ }^{11}$ to fix the regions where dopants can be found.

Another method, presented below, is to fix the $p-i-n$ structure by covalently attaching the dopant ions-in the form of a self-assembled monolayer (SAM) - to one of the interfaces. A similar method has been used to increase the efficiency of small-molecule organic LEDs, ${ }^{12,13}$ but in these cases the effect is attributed to dipolar effects of the SAM and increased wettability of the modified substrate. The effect discussed below differs in that an interfacial dipole layer is insufficient to explain the data, leading to the conclusion that the SAM is electrochemically doping the polymer host.

In this letter, we employ this SAM-based $p-i-n$ structure using a LEC active layer (described below) sandwiched between a Ag anode and SAM-modified indium tin oxide (ITO)

\footnotetext{
${ }^{a)}$ Electronic mail: sacarter@ucsc.edu
}

cathode (Fig. 1). We present quantum efficiency versus time, and current and luminance versus time data on this structure with and without the SAM. We also discuss an analogous LED structure with and without the SAM.

LEC devices were prepared using a $0.8 \mathrm{wt} \%$ solution of the conducting polymer superyellow (SY, Covion/Merck) in chlorobenzene plus $33 \mathrm{wt} \%$ of the polymer electrolyte poly(ethyleneoxide) $\left(5 \times 10^{6}\right.$ molecular weight, PolySciences) and 8 wt $\%$ of the dopant tetra- $n$-butylammonium tetrafluoroborate $\left(\mathrm{TBABF}_{4}, \mathrm{Alfa}\right.$ Aesar), where these last two weight percentages are relative to the polymer. Clean LED devices were also prepared using a $0.5 \mathrm{wt} \%$ solution of SY in chlorobenzene. Formation of the $n$-doping SAM was performed by soaking UV plasma treated substrates in a $5.75 \mathrm{wt} \%$ solution of trimethoxysilylpropyl-tri- $n$-butylammonium chloride $\left(\mathrm{TMS}_{\mathrm{B}} \mathrm{BA}^{+} \cdot \mathrm{Cl}^{-}=\mathrm{TMS} 3 \mathrm{BACl}\right.$, Gelest) in methanol for $24 \mathrm{~h}$ at room temperature. The LED or LEC layer was spun from solution either directly on the ITO surface or on the SAM-modified ITO, then annealed at $140{ }^{\circ} \mathrm{C}$ for $30 \mathrm{~min}$ in vacuum, yielding thicknesses of $110 \mathrm{~nm}$ (LED) and $300 \mathrm{~nm}$ (LEC). A top electrode of Ag was deposited by thermal evaporation at a vacuum of about $10^{-6}$ torr. The final LEC
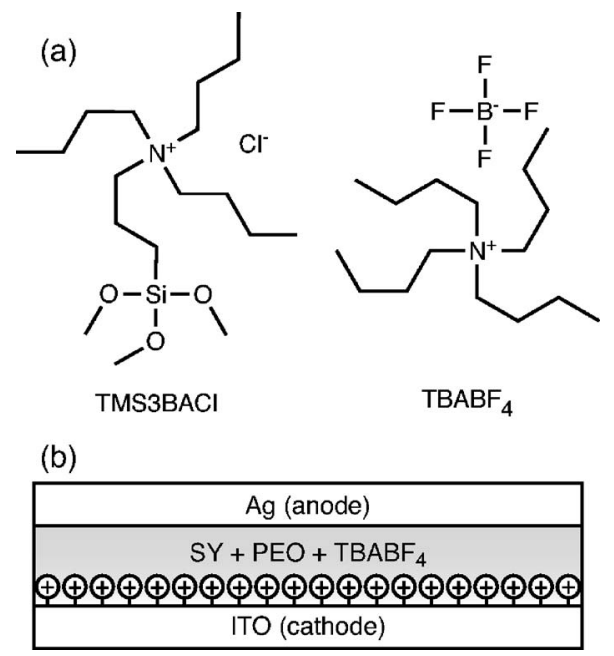

FIG. 1. (a) Chemical structures of TMS3BACl and $\mathrm{TBABF}_{4}$ (note the similarity of the cations). (b) Device structure of SAM-based LEC, where the cation comprising the SAM is TMS3BA ${ }^{+}$. 


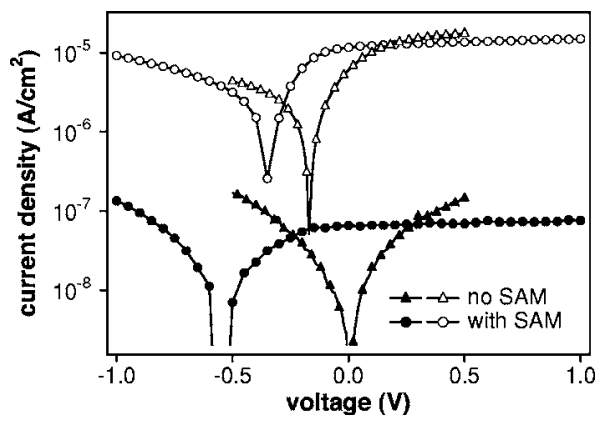

FIG. 2. Current vs voltage data for ITO/SY/Ag (triangles) and ITO/SAM/ SY/Ag (circles). The closed symbols indicate dark current and the open symbols indicate current under solar illumination.

device structure is shown in Fig. 1. Characterization was performed using a Keithley 2400 source meter for current and voltage sourcing and measuring, and a photodiode attached to a Keithley 485 picoammeter to measure electroluminescence. An Oriel xenon arc lamp was used as a solar simulator for photocurrent/photovoltaic analysis. The devices were stored and tested in nitrogen.

As a base line for the effect of the SAM, the LED architectures of ITO/SY/Ag and ITO/SAM/SY/Ag were examined. Typical current versus voltage $(I-V)$ curves in the dark and under solar illumination are shown in Fig. 2; the data are summarized in Table I. Data were obtained by sweeping voltage from $0 \mathrm{~V}$ to some positive voltage, back through $0 \mathrm{~V}$ to the same negative voltage, then back to $0 \mathrm{~V}$ with a sweep rate of $\sim 80 \mathrm{mV} / \mathrm{s}$ (without $\mathrm{SAM}$ ) or $\sim 350 \mathrm{mV} / \mathrm{s}$ (with $\mathrm{SAM}$ ). The positive-to-negative sweeps are shown as they were characteristic of both sweep directions. The data show a strong effect of adding the SAM, i.e., the suppression of the dark current, and increase in open-circuit voltage $V_{\mathrm{oc}}$ in dark and solar illuminated situations. The large $V_{\mathrm{oc}}$ observed in the dark with the SAM-which varied in the range of -300 to $-900 \mathrm{mV}$ between devices and tended to increase slightly with multiple sweeps in rapid succession - can be attributed to migration of the $\mathrm{Cl}^{-}$ions of the TMS3BACl and the slow return of these ions to the SAM interface. Under forward bias, the $\mathrm{Cl}^{-}$ions are forced toward the ITO interface, canceling the charge on the TMS3BA ${ }^{+} \mathrm{SAM}$, and thereby quenching its $n$-doping effect. In reverse bias, the $\mathrm{Cl}^{-}$ions are forced away from the ITO interface, allowing the TMS3BA ${ }^{+}$to $n$ dope the polymer. The observed $V_{\text {oc }}$ in the dark with the SAM is then an artifact of an internal field caused by the arrangement of positive $\left(\mathrm{TMS} \mathrm{BA}^{+}\right)$and negative $\left(\mathrm{Cl}^{-}\right)$charges.

The suppression of current in forward bias, most notably in the dark, is also evidence that the effect of the SAM is not simply to add a dipole layer at the interface. Numerous researchers have investigated the effects of small-molecule

TABLE I. Summary of photovoltaic data for ITO/SY/Ag and ITO/SMA/ SY/Ag structures (as shown in Fig. 2).

\begin{tabular}{llccc}
\hline \hline & Illum. & $V_{\mathrm{oc}}(\mathrm{mV})$ & $I_{\mathrm{sc}}\left(\mu \mathrm{A} / \mathrm{cm}^{2}\right)$ & Fill factor $(\%)$ \\
\hline Without SAM & Solar & -168 & 6.09 & 22 \\
With SAM & Dark & $-550^{\mathrm{a}}$ & 0.0661 & 32 \\
& Solar & -342 & 11.7 & 33 \\
\hline
\end{tabular}

${ }^{\mathrm{a}} \mathrm{SAM}$ dark $V_{\mathrm{oc}}$ varied among different devices in the range of -300 to $-900 \mathrm{mV}$. vices. This may be due to luminescence quenching by a high
Downloaded 16 Mar 2007 to 169.233.74.46. Redistribution subject to AIP license or copyright, see http://apl.aip.org/apl/copyright.jsp

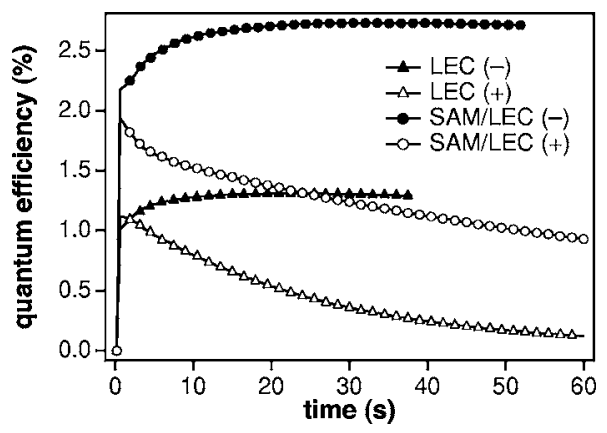

FIG. 3. Quantum efficiency for ITO/LEC/Ag (triangles) and ITO/SAM/ $\mathrm{LEC} / \mathrm{Ag}$ (circles). The + and - signs indicate that the data were taken at constant current density of + or $-3.33 \mathrm{~mA} / \mathrm{cm}^{2}$.

monolayers on the work function of ITO. ${ }^{12-17}$ Unlike the materials chosen by these other researchers, TMS3BACl can become a charged - and therefore doping-species under bias. Using semiempirical Hartree-Fock optimization via the PM3 Hamiltonian, the component of the dipole moment perpendicular to the ITO surface was calculated to be $-6.8 \mathrm{D}$ for TMS3BA $\mathrm{BA}^{+}$and $8.6 \mathrm{D}$ for TMS3BACl, where the perpendicular direction was chosen parallel to the $\mathrm{Si}-\mathrm{N}$ direction (cf. Fig. 1) and pointing away from the ITO. Following the usual electrostatic dipole layer argument, ${ }^{12,18}$ the positive dipole in forward bias, when the $\mathrm{Cl}^{-}$ions are present, should decrease the barrier to hole injection and enhance forward bias current. Instead, current is suppressed, indicating that the SAM's role must be other than a simple dipole layer. Given the argument of the previous paragraph and the structural similarity to the dopant $\mathrm{TBABF}_{4}$ (Fig. 1), the data suggest that the SAM is $n$ doping the polymer in reverse bias.

A final note on the data shown in Fig. 2 is that the consistent negative $V_{\text {oc }}$ indicates that the effective work function arrangement is such that the ITO is the preferred electron injector. While electron injection from ITO is contrary to the standard LED/LEC, ${ }^{5}$ it is consistent within the wide range of reported work functions for ITO [4.3-5.1 eV (Ref. 19)] and $\mathrm{Ag}$ [4.52-4.74 eV (Ref. 20)].

Due to the dynamic nature of the active material, the first experiment that each LEC device was subjected to was constant current stressing, thus "forming" the devices by arranging the internal ions into the $p-i-n$ (or $n-i-p$ ) structure and yielding quantum efficiency $(\mathrm{QE})$ as a function of time. Typical QE versus time for the LEC architecture with and without the SAM is shown in Fig. 3. The devices show a "native" QE before stressing: 2\% with the SAM compared to $1 \%$ without the SAM. On stressing the devices at + or $-3.33 \mathrm{~mA} / \mathrm{cm}^{2}$, both structures show divergence from this native QE. The negative stress (ITO as electron injector) resulted in an increase and stabilization in $\mathrm{QE}$, whereas the positive stress (ITO as hole injector) resulted in the decay of QE toward zero. This behavior indicates that regardless of the SAM, there is a preferred polarity: ITO as cathode and $\mathrm{Ag}$ as anode, similar to the results shown in Fig. 2 for the LED structure. In reverse bias, TMS3BA ${ }^{+}$and $\mathrm{TBA}^{+}$are pushed toward the ITO, both of which $n$ dope the polymer, facilitating electron injection. However, in forward bias, $\mathrm{Cl}^{-}$, as well as $\mathrm{BF}_{4}^{-}$in the LEC, are pushed toward the ITO, quenching the $n$ doping of the SAM. The decay in light emission in forward bias is different from previously reported LECs ${ }^{3,9}$ but was consistently observed for our de- 


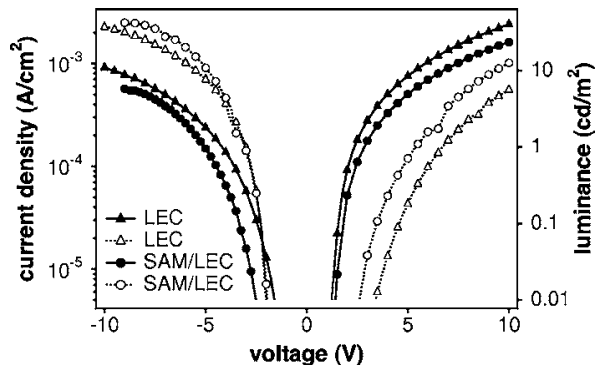

FIG. 4. Current and luminance vs voltage $(I-L-V)$ data for ITO/LEC/Ag (triangles) and ITO/SAM/LEC/Ag (circles). The closed symbols indicate current density, while the open symbols indicate luminance. Note that the data in each bias are from a different set of voltage sweeps on different devices (e.g., the LEC data do not represent a single sweep from -10 to $+10 \mathrm{~V}$, but rather one sweep from -10 to $0 \mathrm{~V}$ and another from +10 to $0 \mathrm{~V}$, on different devices).

concentration of $\mathrm{TBA}^{+}$ions at the $\mathrm{Ag}$ interface; it has been observed that high salt concentration leads to a decrease in electroluminescence. The twofold enhancement in QE is then due to the combined doping of the SAM and the LEC salt. Note that the data presented in Fig. 3 are from the initial current stressing for each of these devices.

Current and luminance versus voltage $(I-L-V)$ data were obtained by sweeping voltage from $0 \mathrm{~V}$ toward some positive or negative voltage (chosen as the point at which the current would reach $\approx 3.33 \mathrm{~mA} / \mathrm{cm}^{2}$ ), back through $0-1 \mathrm{~V}$ of the opposite polarity, then back to zero. In this way, the data represent the $I-L-V$ response of a given forming direction: the reverse bias sweeps represent the case of active $n$ doping at the ITO, whereas forward bias sweeps represent the case of quenched $n$ doping at the ITO. Characteristic LEC $I-L-V$ response is shown in Fig. 4, where the data are from the second of the three sweep directions described above (i.e., with the devices formed). The data indicate that in both biases, the current is lower and luminance higher for the devices with the SAM. In reverse bias, the $n$ doping at the ITO interface not only increases electron injection but also acts as a hole blocking layer. Thus holes attempting to escape into the ITO are blocked at the interface and recombine with injected electrons, increasing light emission but decreasing current. In forward bias, the data suggest that there is a greater imbalance in injected charge, most likely in favor of hole injection from the ITO (given the relative work functions of ITO and Ag), resulting in the noticeable decrease in light emission. As with the LED response (Fig. 2), the presence of the SAM suppresses current. However, the SAM still increases light emission, possibly as a result of continued blocking/balancing of injected charge-even considering the quenched SAM doping.

The LEC devices presented in this letter based on charged SAM-modified ITO electrodes show increased quantum efficiency compared to analogous devices on bare ITO. Similar polymer-only (LED) devices show an increase in open-circuit voltage and short-circuit current under solar il- lumination, leading to a higher photovoltaic fill factor than their bare ITO analogs. These gains are at little expense in that the SAM preparation is easily carried out both in a laboratory environment or in high-throughput production by simple soaking.

These results warrant continued study and optimization of the basic structure. In particular, the SAM material for this research was chosen based on commercial availability and chemical similarity to one of many commonly used LEC dopant ions (Fig. 1). Other SAM materials, forming layers at either-or both — of the polymer/electrode interfaces could be feasibly employed. Furthermore, the SAM material presented above has only a propyl link $(\lesssim 1 \mathrm{~nm})$ between the silane head group and charged tail group. One interesting possibility would be to use SAM materials-or polymeric materials-with a significantly longer link, providing fixed $n$ and/or $p$ doping deeper into the bulk of the device.

The authors wish to thank Jian Ping Chen for his assistance throughout this project. This research was funded by a Small Business Technology Transfer Grant from the National Science Foundation (USA, Award No. 0539802) in conjunction with Add-Vision, Inc., Scotts Valley, CA.

${ }^{1}$ Q. Pei, G. Yu, C. Zhang, Y. Yang, and A. J. Heeger, Science 269, 1086 (1995).

${ }^{2}$ L. Edman, Electrochim. Acta 50, 3878 (2005).

${ }^{3}$ Q. Pei, Y. Yang, G. Yu, C. Zhang, and A. Heeger, J. Am. Chem. Soc. 118, 3922 (1996).

${ }^{4}$ J. H. Burroughes, D. D. C. Bradley, A. R. Brown, R. N. Marks, K. Mackay, R. H. Friend, P. L. Burns, and A. B. Holmes, Nature (London) 347, 539 (1990).

${ }^{5}$ R. H. Friend, R. W. Gymer, A. B. Holmes, J. H. Burroughes, R. N. Marks, C. Taliani, D. D. C. Bradley, D. A. D. Santos, J. L. Bredas, M. Logdlund, and W. R. Salaneck, Nature (London) 397, 121 (1999).

${ }^{6}$ J. Gao, G. Yu, and A. J. Heeger, Appl. Phys. Lett. 71, 1293 (1997).

${ }^{7}$ L. Edman, M. A. Summers, S. K. Buratto, and A. J. Heeger, Phys. Rev. B 70, 115212 (2004).

${ }^{8}$ J. Dane, C. Tracy, and J. Gao, Appl. Phys. Lett. 86, 153509 (2005).

${ }^{9}$ J. Gao, Y. Li, G. Yu, and A. J. Heeger, J. Appl. Phys. 86, 4594 (1999).

${ }^{10}$ J.-H. Shin, S. Xiao, A. Fransson, and L. Edman, Appl. Phys. Lett. 87, 043506 (2005)

${ }^{11}$ T.-W. Lee, H.-C. Lee, and O. O. Park, Appl. Phys. Lett. 81, 214 (2002).

${ }^{12}$ S. F. J. Appleyard, S. R. Day, R. D. Pickford, and M. R. Willis, J. Mater. Chem. 10, 169 (2000).

${ }^{13}$ J. Lee, B.-J. Jung, J.-I. Lee, H. Y. Chu, L.-M. Do, and H.-K. Shim, J. Mater. Chem. 12, 3494 (2002).

${ }^{14}$ N. Karsi, P. Lang, M. Chehimi, M. Delamar, and G. Horowitz, Langmuir 22, 3118 (2006).

${ }^{15}$ E. Bruner, N. Koch, A. Span, S. Bernasek, A. Kahn, and J. Schwartz, J. Am. Chem. Soc. 124, 3192 (2002).

${ }^{16}$ R. A. Hatton, S. R. Day, M. A. Chesters, and M. R. Willis, Thin Solid Films 394, 291 (2001).

${ }^{17}$ W. Osikowicz, X. Crispin, C. Tengstedt, L. Lindell, T. Kugler, and W. R. Salaneck, Appl. Phys. Lett. 85, 1616 (2004).

${ }^{18}$ H. Ishii, K. Sugiyama, E. Ito, and K. Seki, Adv. Mater. (Weinheim, Ger.) 11, 605 (1999).

${ }^{19}$ J. S. Kim, B. Lagel, E. Moons, N. Johansson, I. D. Baikie, W. R. Salaneck, R. H. Friend, and F. Cacialli, Synth. Met. 111-112, 311 (2000).

${ }^{20}$ Handbook of Chemistry and Physics, 87 th ed., edited by D. R. Lide (CRC, Boca Raton, FL, 2006), p. 12.114 